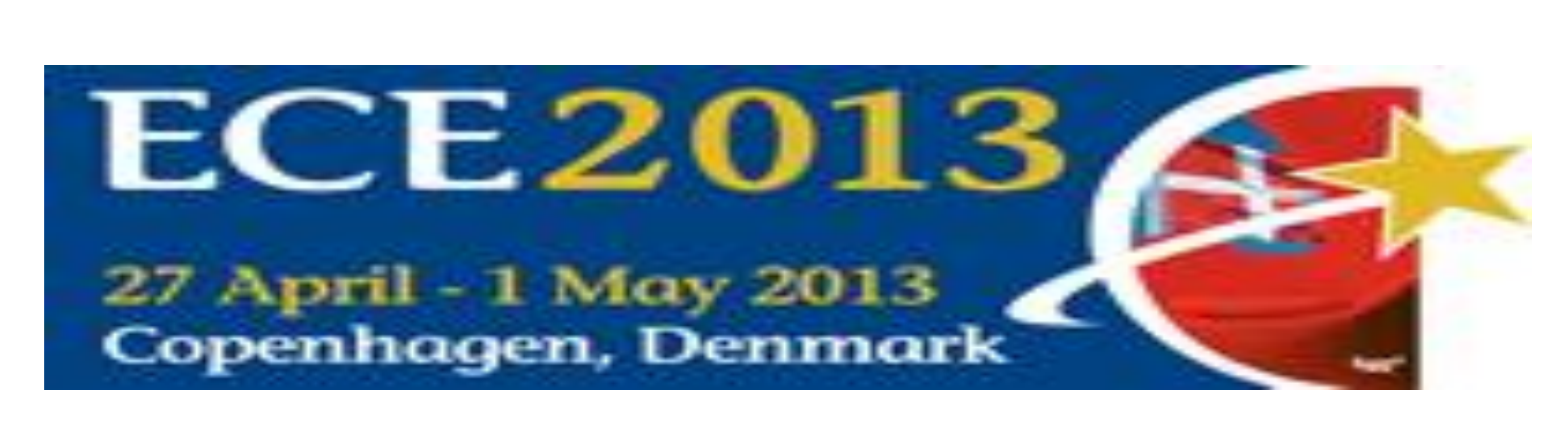

\title{
Hyperaldosteronism in patients with Hyperparathyroidism: 3 cases
}

\author{
T. Ruiz Gracia, F. Fernández Capel , E. Gómez Hoyos, M. Cuesta Hernández , N. Fuertes Zamorano, A. Calle Pascual,
} I. Runkle de la Vega. Endocrinology Service. Hospital Clínico San Carlos, Madrid. Spain

\section{Introduction:}

Hyperaldosteronism may induce elevated parathyroid hormone (PTH) levels, presumably by increasing urinary and fecal losses of $\mathrm{Ca}^{2+}$ and $\mathrm{Mg}^{2+}$. Therefore, this dyshomeostatic balance leads to secondary hyperparathyroidism. Furthermore, PTH stimulates in vitro the secretion of aldosterone in a concentration dependent manner, and increases angiotensin-IIstimulated aldosterone release (by dehydration). Recentely, PTH receptors had been described in aldosteronoma tissue in a patient with hyperaldosteronism and hyperparathyroidism.

\section{Material and Methods:}

We present three patients who were sent to an endocrinologist for treatment and follow-up of primary hyperparathyroidism, with resistant hypertension, and in whom hyperaldosteronism was diagnosed.

Aldosterone (Ald) and Renin (re) (RIA) are expressed in $\mathrm{pg} / \mathrm{ml}$. The captopril test (CAP) was performed per protocol: minimum of two weeks on doxazosin as sole antihypertensive, minimum $133 \mathrm{mEq}$ sodium intake for three days, basal Ald/re (BAld/re), 1 and 2 hours post$25 \mathrm{mg}$ captopril. Test is positive if after 2 hours ald $>130$ or Ald/re > 50 .

\section{Results:}

CASE 1: 74 year old male remitted to follow up of primary hyperparathyroidism. Office BP: 160/95 mmHg, on losartan (100 mg), amlodipine $(10 \mathrm{mg})$, hydrochlorothiazide $(25 \mathrm{mg})$, atenolol $(50 \mathrm{mg})$. The ultrasound examination revealed: parathyroid adenoma located in the left thyroid lobe. He rejects parathyroid surgery. He rejects adrenal catheterization/surgery. Treatment: eplerenone $50 \mathrm{mg}$ b.i.d, cinacalcet $30 \mathrm{mg}$ q.d.

CASE 2: 68 year old female referred following parathyroidectomy for parathyroid hyperplasia. Office BP: $200 / 100$ mmHg, on losartan $(50 \mathrm{mg})$, hydrochlorothiazide $(12.5 \mathrm{mg})$, atenolol $(50 \mathrm{mg})$. The patient rejects catheterization/surgery. Treatment: eplerenone $50 \mathrm{mg}$ b.i.d.

CASE 3: 81 year old female, referred from rheumatology for primary hyperparathyroidism secondary to vitamin D deficit. Office BP: $175 / 90 \mathrm{mmHg}$, on nebivolol $(5 \mathrm{mg})$, lercardipine $(20 \mathrm{mg})$, furosemide (40 $\mathrm{mg}$ ). Parathyroid disease not detected by ultrasound.

Treatment: spironolactone $100 \mathrm{mg}$ q.d, cinacalcet $30 \mathrm{mg}$ q.d.

\begin{tabular}{|c|c|c|c|c|c|c|c|c|c|c|c|}
\hline & $\begin{array}{c}\text { Basal Ca } \\
(\mathrm{mg} / \mathrm{dl})\end{array}$ & $\begin{array}{c}\text { Basal PTH } \\
(\mathrm{mg} / \mathrm{dl})\end{array}$ & $\begin{array}{c}25 \text { OH Vit D } \\
(\mathrm{ng} / \mathrm{ml})\end{array}$ & $\begin{array}{l}\text { Initial K } \\
\text { (mmol/L) }\end{array}$ & $\begin{array}{l}\text { Inicial office BP } \\
(\mathrm{mmHg})\end{array}$ & $\begin{array}{l}\text { Antihyp. } \\
\text { Drugs }\end{array}$ & $\begin{array}{c}\text { Screen } \\
\text { Ald/re (pg/ml) }\end{array}$ & $\begin{array}{c}\text { CAP: } \\
\text { Basal Ald/re }\end{array}$ & $\begin{array}{c}\text { CAP: } \\
\text { 1h Ald/re }\end{array}$ & $\begin{array}{c}\text { CAP: } \\
\text { 2h Ald/re }\end{array}$ & $\begin{array}{l}\text { Post-treat. } \\
\text { OBP mmHg) }\end{array}$ \\
\hline Case 1 & 11.1 & $\begin{array}{c}71 \\
\text { IRMA }\end{array}$ & $\begin{array}{l}44.7 \\
\mathrm{ChL}\end{array}$ & $\begin{array}{c}3.8 \\
(4.4)^{*}\end{array}$ & 160/95 & 4 & $\begin{array}{l}293 / 2 \\
(146)\end{array}$ & $\begin{array}{c}352 / 3 \\
(117.3)\end{array}$ & $\begin{array}{c}339 / 2 \\
(169.5)\end{array}$ & $\begin{array}{l}408 / 3 \\
(136)\end{array}$ & $110 / 70$ \\
\hline Case 2 & 11.4 & $\begin{array}{c}84 \\
\text { IRMA }\end{array}$ & $\begin{array}{l}47.7 \\
\text { ChL }\end{array}$ & 3.8 & $200 / 100$ & 3 & $\begin{array}{c}171 / 3 \\
(57)\end{array}$ & $\begin{array}{l}174 / 4 \\
(43.5)\end{array}$ & $\begin{array}{l}222 / 4 \\
(55.5)\end{array}$ & $\begin{array}{c}170 / 5 \\
(34)\end{array}$ & $126 / 85$ \\
\hline Case 3 & 10.9 & $\begin{array}{l}129 \\
\mathrm{ChL}\end{array}$ & $\begin{array}{l}53.5 \\
\text { ChL }\end{array}$ & 4 & $175 / 90$ & 3 & $\begin{array}{c}204 / 3 \\
(68)\end{array}$ & $\begin{array}{c}203 / 2 \\
(101.5)\end{array}$ & $\begin{array}{l}129 / 6 \\
(21.5)\end{array}$ & $\begin{array}{l}169 / 9 \\
(18.7)\end{array}$ & $110 / 70$ \\
\hline
\end{tabular}
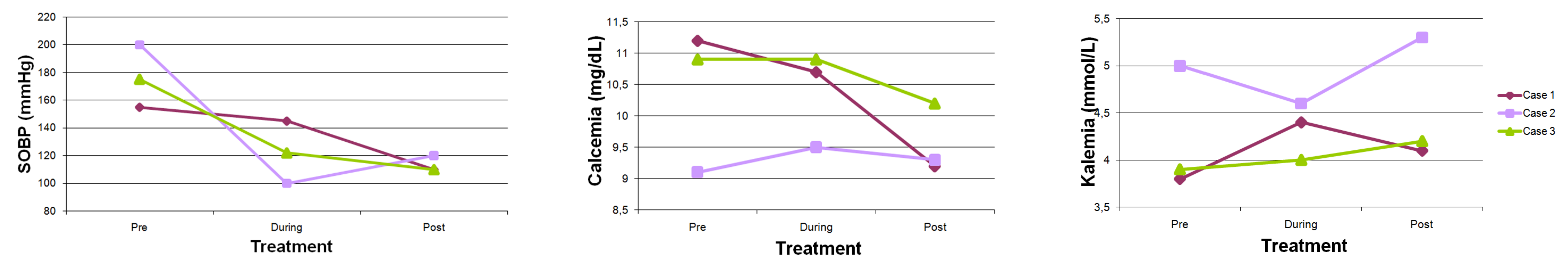

K: Kalemia. *Kalemia on cinacalcet. Ca: Calcemia. PTH: Parathyroid hormone. ChL: Chemiluminescence. SOBP: Systolic office blood pressure. Antihyp: Antihypertensive. Screen: Screening. Ald: Aldosterone. re: Renin. CAP: Captopril Test (25 mg)

\section{Conclusion:}

Three cases of hyperaldosteronism associated with hyperparathyroidism were detected in the clinic of a single endocrinologist over 2 years, suggesting that the association is not infrequent, and underlying the importance of ruling out hyperaldosteronism in patients with hyperparathyroidism and moderate severe or resistant hypertension. 